\begin{tabular}{|c|l|}
\hline Title & Improved accuracy of computer-assisted cervical pedicle screw insertion \\
\hline Author(s) & Kotani, Y oshihisa; A bumi, Kuniyoshi; Ito, Manabu; Minami, A kio \\
\hline Citation & Journal of Neurosurgery : Spine, 99(3), 257-263 \\
\hline Issue Date & 2003-10 \\
\hline Doc URL & http://hdl.handle.net/2115/14767 \\
\hline Rights & $\begin{array}{l}\text { The final version of the paper was published in Journal of Neurosurgery:Spine, vol. 99-3. For reuse of any of the } \\
\text { materials, including editorial copy, figures, or tables please contact the Journal of Neurosurgery atjneuro@ virginia.edu }\end{array}$ \\
\hline Type & article (author version) \\
\hline File Information & JN99-3.pdf \\
\hline
\end{tabular}

Instructions for use 


\title{
IMPROVED ACCURACY OF COMPUTER-ASSISTED CERVICAL PEDICLE SCREW INSERTION
}

\author{
Yoshihisa Kotani, M.D.*, Kuniyoshi Abumi, M.D.*, \\ Manabu Ito, M.D.*, and Akio Minami, M.D.* \\ *: Dept. of Orthopaedic Surgery, Hokkaido University Graduate School of Medicine, Kita-15, \\ Nishi-7, Kitaku, Sapporo 060-8638 JAPAN, \\ Corresponding author: Yoshihisa Kotani,M.D. \\ Dept. of Ortho. Surg., Hokkaido University Graduate School of Medicine \\ Kita-15, Nishi-7, Kitaku, Sapporo 060-8638, Japan \\ Fax:81-11-706-6054, E-mail:y-kotani@med.hokudai.ac.jp
}

Running Head: Computer-assisted cervical pedicle screw insertion 


\section{Abstract}

Object. The authors introduced a unique computer-assisted cervical pedicle screw (CPS) insertion technique that was specially modified by original pedicle screw insertion instruments. The accuracy of screw placement as well as surgical outcome and complication rates were compared between a computer-assisted and conventional manual insertion techniques.

Methods. A screw insertion guiding system consisted of a modified awl, probe, tap and a screwdriver that were specially designed for a computer-assisted cervical pedicle screw insertion. Using this system, a real-time instrument/screw tip information was three-dimensionally identified in each step of screw insertion. Seventeen patients underwent a cervical pedicle screw fixation using a computer-assisted surgical navigation system. The cervical disorders consisted of spondylotic myelopathy with segmental instability or kyphosis, metastatic spine tumor, rheumatoid spine, postlaminectomy kyphosis and etc. The pedicle wall perforation rate was significantly lower in computer-assisted group than that in manual insertion group $(1.2 \%$ vs $6.7 \%)(\mathrm{P}<0.05)$. The screw trajectory in the horizontal plane was significantly closer to the anatomical pedicle axis in computer-assisted group when compared to the manual insertion group $(\mathrm{P}<0.05)$. The increased screw trajectory significantly reduced the incidence of screw perforation laterally. The clinical complication such as neural damage or vascular injury was not demonstrated in computer-assisted group 
(0\% vs $2 \%)$. The overall surgical outcome was satisfactory.

Conclusions. . In contrast to the previously reported computer-assisted technique, our unique computer-assisted CPS insertion technique provides the real-time and three-dimensional instrument/screw tip information. This serves as a powerful tool for the safe and accurate pedicle screw placement in the cervical spine.

Key words: Computer-assisted surgery, Cervical pedicle screw fixation, Cervical spine 


\section{Introduction}

With the recent progression of surgical navigation technologies, the basic and clinical reports regarding a spinal navigation surgery are increasing. ${ }^{5-18}, 21,24$ The computer-assisted surgery in the spine is presently applied to a neural decompression, tumor resection, a visualization of surrounding tissues such as great vessels, vertebral arteries, and other vital organs, and a spinal instrumentation surgery. In spinal instrumentation procedure, the real-time imaging of instrument positioning has been performed mainly in thoracolumbar and lumbar pedicle screw placement. ${ }^{10,13,14,16,17}$

There have been a very few reports regarding an application of computer-assisted surgery to a cervical spinal instrumentation. Weidner et al. utilized a surgical navigation system for transarticular screw fixation at C1-2 in thirty-seven patients, and reported a decreased frequency of screw misplacement. ${ }^{24}$ Bolger et al. demonstrated a efficacy of surgical navigation system in thirty-four cases of C1-2 transarticular screw and fifteen cases of lateral mass screw fixation. ${ }^{6}$ In turn, the clinical series of cervical pedicle screw fixation have been reported by Abumi et al., demonstrating an efficacy in the treatment of unstable cervical disorders and a correction of deformity. ${ }^{1,2,3,4}$ To date, we have experienced over 300 clinical cases of cervical pedicle fixation and found a risk of screw insertion in cases with small pedicles in a rheumatoid spine and pediatric patients, cervical deformity, anomalous pedicles with asymmetry, and a unilateral vertebral artery occlusion. ${ }^{1,3}$ In order to perform a 
cervical pedicle screw fixation safely in those high risk cases, we started to use a surgical navigation system clinically from early 2001.

In this manuscript, we introduced a unique screw insertion technique for a computer-assisted cervical pedicle screw insertion that was modified by original pedicle screw instruments. Secondary, a comparative accuracy of screw placement as well as surgical outcome and complication rates were investigated between a computer-assisted and manually inserted cases. 


\section{Materials and Methods}

\section{Computer-assisted pedicle screw insertion}

We utilized a surgical navigation system (StealthStation, SofamorDanek, Memphis, TN)

combined with our unique screw insertion instruments for cervical pedicle screws. The

navigation system consisted of a computer system and a charge-coupled device (CCD)

camera, allowing CT data to convert into the hard disk and reconstruct three-dimensional

spinal image preoperatively. The reference frame was attached to a spinous process of

vertebra, allowing its signal to be sent to CCDcamera. The subsequent registration

procedures of point-merge and surface-merge produced three-dimensional spinal images on

the monitor, which were identical to the actual spines in the operative field. Instead of solely

using a conventional navigation probe, our unique screw insertion instruments consisted of a

modified awl, probe, tap and screwdriver only for cervical pedicle screw use (Figure 1).

These instruments were modified from original pedicle screw instruments, however, special

considerations were required to prevent a bending of instrument during insertion as well as

to attach to the image guided awl/probe/tap driver (Figure 1). The tip configuration of these

instruments was also modified for cervical use. The pharmaceutical affairs law and new

design for practical use in Japan approved this system. Using this system, the

instrument/screw tip information was three-dimensionally identified in each step of screw

insertion (Figure 2). Therefore, surgeons can assure a three-dimensional position of 
instrument/screw tip in the axial, midsagittal, parasagittal, and coronal images during the operation procedure.

\section{Patient demographics}

From March 2001, seventeen patients underwent a cervical pedicle screw fixation using a computer-assisted technique. There were seven males and ten females. The average age was forty-two years old (6-83 years old). The cervical disorders were spondylotic myelopathy with segmental instability or kyphosis in five, metastatic spine tumor in three, rheumatoid spine in three, postlaminectomy kyphosis in two, simultaneous spinal reconstruction for spinal cord tumor in two, os odontoideum and congenital cervicothoracic scoliosis in one respectively. The stabilized spinal segments were 3.7 segments in average (1-6 segments). A total of 78 pedicle screws were inserted using a computer-assisted technique from C2 to C7. The screw diameter was mostly $3.5 \mathrm{~mm}$, however, three cases allowed $4.0 \mathrm{~mm}$ screws. The mini VSP plate (Depuy AcroMed, Rayham, MA) was used in five cases, while 3/16 inch Isola rods in twelve cases. The combined surgeries were laminectomy or laminoplasty in eleven, tumor resection in two, foraminotomy in two, and posterior resection of hemivertebra and unsegmented bar in one. The bone graft was conducted with iliac bone in five and local bone in twelve. 


\section{Radiologic analysis of pedicle screw placement}

The radiographic evaluation was conducted in terms of a pedicle wall perforation of screws and a screw trajectory in the axial image of CT scan. Using lateral and oblique x-rays, the screw perforation to upper or lower direction was identified and recorded. The medial or lateral perforation of screws from the pedicle wall was also evaluated. The abnormality of screw placement in length was also recorded when screw penetrated the anterior wall of vertebral body. Regarding a pedicle wall perforation, manually operated 180 cases were retrospectively examined using x-rays and CTs, and served as a manually inserted perforation rate. In terms of the screw trajectory, our recent series of 50 manual cases were retrospectively examined and served as manual data. These data with manual insertion technique were statistically compared using Fisher's exact test for pedicle perforation rates and one-way analysis of variance (ANOVA) with post-hoc analysis of StudentNewman-Keuls test for screw trajectory.

\section{Clinical outcome and complications}

The neurologic recovery and the change of ambulatory status were examined preoperatively and postoperatively. The complications directly or indirectly related to the surgery were recorded. The manually operated 180 cases were retrospectively examined and compared to the computer-assisted cases in terms of complication rate. We focused on an indirect or 
direct nerve injury, vascular injury, instrumentation failures and other neural disturbances related to the surgery. The data was statistically compared using a Fisher's exact test. 


\section{Results}

\section{Computer-assisted pedicle screw insertion}

Using a computer-assisted screw insertion guiding system, we successfully inserted the cervical pedicle screws even in pediatric, deformity or severely spondylotic cases. In a navigation monitor, actual instrument tip position was identified in axial, sagittal and coronal images (Figure 2).

\section{Radiologic analysis of pedicle screw placement}

The pedicle wall perforation on x-rays and CT was identified in one screw out of 78 screws inserted (1.2\%). The case with a perforation showed a slight lateral breach of C5 pedicle screw out of pedicle wall, however, no neurovascular complication occurred. The retrospective analysis of 180 cases demonstrated the pedicle wall perforation of 45 screws out of 669 screws (6.7\%). The perforation rate of computer-assisted insertion (1.2\%) was statistically lower than that of manual insertion in 180 cases $(6.7 \%)(\mathrm{P}<0.01)$.

The pedicle screw trajectory in the horizontal plane differs among anatomical, computer-assisted, and manual insertion groups (Figure 3). In terms of anatomical angles, Panjabi et al. reported a quantitative three-dimensional anatomical data obtained from human cadaveric cervical spines. ${ }^{19}$ The representative angles on the horizontal planes were 42.9, 43.9, 41.2, 34.1, and 26.7 degrees in average at C3, C4, C5, C6, and C7 levels, respectively. 
Our manual screw angles from fifty patients ranged from 24 degrees to 27 degrees at C3-7.

When using a computer-assisted surgery, the average screw angles were 30, 30.6, 34, 27.5, and 32 degrees at C3, C4, C5, C6, and C7 levels, respectively. At C5 level, the angle in the computer-assisted group demonstrated a significantly larger value than that in manual insertion group $(\mathrm{P}<0.05)$. The statistical difference between anatomical and manual insertion group was also detected at C5 level $(\mathrm{P}<0.05)$. At C3, C4 and C6 levels, the anatomical angles showed significantly higher values than those in the computer-assisted and manual insertion groups $(\mathrm{P}<0.05)$.

\section{Clinical outcome and complications}

The clinical follow-up periods were fifteen months in average (six to 22 months). In all cases with a computer-assisted surgery, a preoperative myelopathy and radiculopathy recovered and all patients ambulated after surgery. The preoperative kyphosis of 44 degrees in postlaminectomy kyphosis patients corrected to one degree in average postoperatively. In congenital cervicothoracic scoliosis patient, preoperative scoliosis of 40 degrees corrected to 12 degrees postoperatively. There were no vascular and neural complications in computer-assisted surgeries. In previous review of 180 manual cases, there were two cases of direct nerve root injury (1\%), one case of indirect nerve root injury (0.5\%) and one case of vertebral artery injury $(0.5 \%){ }^{1,3}$ The indirect nerve injury was caused by foraminal stenosis 
following a kyphosis correction. The statistical comparison of complication rate between computer-assisted group and manual group did not reach a significance.

\section{Case Presentations}

Case1 (Figure 4)

A 17-year-old male underwent a posterior decompression surgery from an occicital bone to C3 by former doctor due to Chiari malformation. At four months postoperatively, a kyphotic deformity from C2 to C5 increased gradually with an intractable neck pain. At the initial consultation to our department, there were severe kyphosis from C2 to C5 and morphologic asymmetry in bilateral cervical pedicles (Figure 4A-E). MR angiography demonstrated a possible occulusion of left vertebral artery. The corrective surgery was safely conducted with a surgical navigation system (Figure 4F). The posterior correction and stabilization from C2 to C5 was done using a cervical pedicle screw system. At one year postoperatively, the fusion is complete without any correction loss and instrumentation failure.

Case 2 (Figure 5)

A 52-year-old male presented a progressive gait disturbance and paralysis of both hands due to metastatic cervical spine tumor originated from the prostate carcinoma. Radiographs and MRI scans demonstrated the multiple metastases on whole cervical spines and a kyphotic deformity with spinal cord compression. Posterior spinal stabilization was performed from 
C3 to T1 level with the assistance of navigation system, followed by anterior decompression and fibula graft. At 18 months postoperatively, bony fusion was obtained completely and a stable spine was preserved. Postoperative CT scans demonstrated the correct positioning of screws.

Case 3 (Figure 6)

A 68-year-old male presented a severe cervical myelopathy due to cervical spondylosis with kyphosis and a segmental instability at C3/4. The preoperative kyphosis was 22 degrees at C3 to C5. The posterior correction and fusion was performed using a pedicle screw system from C3 to C5. The postoperative kyphosis was corrected to 6 degrees and maintained until the completion of fusion. 


\section{Discussion}

In terms of the application of computer-assisted technology to cervical instrumentation surgery, there have been a few reports demonstrating clinical results of C1-2 transarticular screw fixation, ${ }^{6,24}$ however, there is a paucity of information regarding an efficacy of computer-assisted surgery in cervical lateral mass or pedicle screw fixation. Richter et al. experimentally investigated a validity of computer navigation system for the cervical pedicle screw fixation in cadaveric spines. ${ }^{20}$ Consequently ninety-two percent of screw placement was accurate, demonstrating a clear advantage of navigation surgery. Bolger et al. clinically reported an effectiveness of navigation system in fifteen cases of rheumatoid cervical spines and tumor reconstructions using lateral mass screw fixation. ${ }^{6}$

They emphasized on the increased accuracy of screw placement and usefulness on preoperative surgical planning. The present study serves as a first study comparing a surgical accuracy and clinical complications between navigation surgeries and manually operated cases in a large number of patients. The study results demonstrated that the computer-assisted cervical pedicle screw fixation was more accurate than the manual screw insertion as well as showing lower complication rates.

In contrast to several reports demonstrating an advantage of navigation system in accuracy improvement, there have been some criticisms in the effectiveness of this system. Ludwig et al. experimentally compared the accuracy of screw placement between manual 
insertion and computer-assisted surgery, demonstrating no significant improvement in computer-assisted group. ${ }^{15}$ However, in previous experimental and clinical reports, there is a clear technical issue which does not improve the accuracy in screw placements. ${ }^{12}$ In previous technique, the instrument guide tube with light-emitting diode (LED) was navigated only on the surface of lamina, and the actual tip of awl, tap, and screws were not directly visualized on the navigation monitor in each step of screw insertion. The screw insertion point and angulations of instruments were only verified in this technique. Our unique screw insertion technique for cervical pedicle screw insertion provided accurate, three-dimensional and real-time instrument tip information, serving as an effective tool for the safe and reliable pedicle screw placement in the cervical spine. This technique effectively prevents a lateral screw perforation at the final step of screw insertion after screw tapping procedure in osteoporotic or rheumatoid patients. Manual screw insertion into the pedicle of cervical vertebra with severe deformities or degenerative changes accompanies high risks for neurovascular structures. Our computer-assisted guiding technique enables us to insert a pedicle screw even in such cases, and provides more effective results of reconstructive surgery in the cervical spine.

There are several limitations in this study. First, the number of our operative cases with surgical navigation system is still small and preliminary. The large consecutive cases are required to compare two groups reliably. Secondly, there is still an unsolved problem in 
evaluating a screw perforation accurately. In this study, we utilized AP and oblique X-ray films and CT scans routinely with an occasional 3-D CT scan. The CT image produces a metal artifact around screws, sometimes exaggerating a screw outline. In our experience, a multiplannar 3-D CT scan is most effective in detecting a minor perforation of screw placement. However, the definite standard and methodology of assessing the screw perforation is expected in the future.

Importantly, the risk of screw perforation can be reduced using a navigation system, however, it did not disappeared completely. Although the lateral perforation of CPS did not directly lead to vertebral artery injury, its injury on the dominant side can cause the cerebellar infarction and possibly a fatal sequel. ${ }^{3,22,23}$ To prevent these injuries, the preoperative evaluation of vertebral artery and planning of screw insertion are indispensable. The screw insertion for pedicle diameter below 3.5mm should be avoided even when using navigation assistance.

Considering the present status of spinal navigation surgery, there are several problems requiring a further modification and developments in technology. First, an operation space becomes narrower and additional operating staffs are required for navigation system. Secondary, the registration procedure requires five to ten minutes for each vertebra when using a CT-based navigation system. We utilized a navigation system for the limited vertebra with small pedicles or anomaly and saved the operation time. Thirdly, the 
preoperative CT data acquisition and surgical planning on the computer are required. The fluoronavigation system does not require the preoperative data acquisition to the computer, however, the overall accuracy of the system does not allow the practical use in cervical spine. The recently developed mobile CT scan has a large possibility in the future computer-assisted surgery. Forth, in the present navigation system, the operators have to look away from the operative field and look at the monitor in a screw insertion. Although the learning curve can improve the safety of this procedure, we require the data fusion technology providing operative and imaginary views simultaneously before our eyes in the near future.

Finally, even through the preliminary experience of computer-assisted surgery, we are aware of large future possibility in this technique. In present series, previous C-arm based insertion technique did not allow the screw insertion especially in scoliosis, severe spondylosis, or pediatric cases. The new computer-assisted technique clearly expands the use of cervical pedicle screws in such cases as well as minimizing several risks of complex reconstructive surgery in cervical and cervicothoracic spines. Further modifications and developments in technology are expected for patient safety and more advanced surgical techniques. 


\section{Conclusion}

The accuracy of cervical pedicle screw placement using a unique computer-assisted technique as well as a frequency of complication rate was clinically analyzed when compared to conventional manual insertion technique. The computer-assisted technique provided the accurate three-dimensional instrument tip information, serving as an effective tool for the safe and reliable pedicle screw placement in the cervical spine.

\section{Acknowledgement}

We would like to acknowledge Toshiharu Yamada and Tetsuya Takano in Medtronic Sofamordanek Japan, Co. LTD, Osaka, Japan for the development and technical support of cervical computer-assisted instruments as well as Itaru Oda for the assistance of several experimental investigations. 


\section{References}

1. Abumi K, Ito M, Kotani Y. Complications of cervical pedicle screw placement. Seminars in spine surgery 14:112-124, 2002.

2. Abumi K, Kaneda K. Pedicle screw fixation for non-traumatic lesions of the cervical spine. Spine 22:1853-1863, 1997.

3. Abumi K, Shono $\mathrm{Y}$, Ito $\mathrm{M}$, et al. Complication of pedicle screw fixation in reconstructive surgery of the cervical spine. Spine 25:962-969, 2000.

4. Abumi K, Shono Y, Taneichi T, et al. Correction of cervical kyphosis using pedicle screw fixation systems. Spine 24:2389-2396, 1999.

5. Albert JT, Klein RG, Vaccaro RA. Image guided anterior cervical corpectomy. Spine 24:826-830, 1999.

6. Bolger C, Wigfield C. Image-guided surgery: applications to the cervical and thoracic spine and and a review of the first 120 procedures. J Neurosurg (Spine 2) 92:175-180, 2000.

7. Foley KT, Smith MM. Image-guided spine surgery. Neurosurgery clinics of North America 7:171-186, 1996.

8. Glossop DN, Hu WR, Randle AJ. Computer-aided pedicle screw placement using frameless stereotaxis. Spine 21:2026-2034, 1996. 
9. Holly LT, Bloch O, Obasi C, et al. Frameless stereotaxy for anterior spinal procedures. J Neurosurg (Spine 2) 92:175-180, 2000.

10. Kamimura M, Ebara S, Itoh H, et al. Accurate pedicle screw insertion under the control of a computer-assisted image guiding system: Laboratory test and clinical study. J Ortho Science 4:197-206, 1999.

11. Klein GR, Ludig SC, Vaccaro AR, et al. The efficacy of using an image-guided kerrison punch in performing an anterior cervical foraminotomy. Spine 24:1358-1362, 1999.

12. Kotani Y, Abumi K, Ito M, et al. Improved accuracy of cervical pedicle screw fixation using a newly developed computer-assisted insertion technique. Presented at $18^{\text {th }}$ annual meeting of Cervical Spine Research Society (Europian section), June 13-14, Paris, France.

13. Laine T, Schlenzka D, Makitalo K, et al. Improved accuracy of pedicle screw insertion with computer-assisted surgery. A prospective clinical trial of 30 patients. Spine 22:1254-1258, 1997.

14. Laine T, lund T, Ylikoski M, et al. Accuracy of pedicle screw insertion with and without computer assistance: a randomized controlled clinical study in 100 consecutive patients. Eur Spine J 9:235-240, 2000.

15. Ludwig SC, Kowalski JM, Edwards CC, et al. Cervical pedicle screws. Comparative accuracy of two insertion techniques. Spine 25, 2675-2681, 2000. 
16. Merloz P, Tonetti J, Pittet L, et al. Computer-assisted spine surgery. Computer Aided Surgery 3:297-305, 1998.

17. Merloz P, Tonetti J, Pittet L, et al. Pedicle screw placement using image guided techniques. Clin Orthop 354:39-48, 1998.

18. Ohmori K, Kawaguchi Y, Kanamori M, et al. Image-guided anterior thoracolumbar corpectomy. A report of three cases. Spine 26:1197-1201, 2001.

19. Panjabi MM: Cervical human vertebrae, Quantitative three-dimensional anatomy of the middle and lower regions. Spine 16:861-69, 1991.

20. Richter M, Amiot L, Neller S, et al. Computer-assisted surgery in posterior instrumentation of the cervical spine: an in vitro feasibility study. Eur Spine J 9:S65-S70, 2000.

21. Schlenzka D, Laine T, Lund T. Computer-assisted spine surgery. Eur Spine J 9 (Suppl 1):S57-64, 2000.

22. Shintani A, Zervas NT. Consequence of ligation of the vertebral artery. J Neurosurg 36:447-50, 1972.

23. Smith MD, Emery SE, Dudley A, Murray KJ, Leventhal M. Vertebral artery injury during anterior de4compression of the cervical spine. A retrospective review of ten patients. J Bone Joint Surg Br. 75:410-5, 1993.

24. Weidner A, Wahler M, Chiu ST, et al. Modification of C1-2 transarticular screw fixation 
by image-guided surgery. Spine 25:2668-2674, 2000. 
Figure Legends

Figure 1. Screw insertion guiding system specially designed for cervical pedicle screws.

The system consisted of modified awl, probe, tap and screwdriver that were modified from original pedicle screw instruments. The special considerations were required to prevent a bending of instrument during an insertion as well as to attach them to the image guided awl/probe/tap driver (SofamorDanek, Memphis, TN). Each instrument was simply attached to the image guided awl/probe/tap driver without re-registration procedures.

Figure 2. Cervical pedicle screw insertion with simultaneous and three-dimensional instrument/screw tip information

A: Navigation views provide horizontal, parasagittal and pedicle-axis images in conjunction with a planned screw and actual instrument tip image.

B: The reference frame was attached to the spinous process of corresponding vertebra for spinal navigation. The optical signal from LED on the image guided driver was transmitted to the computer and the instrument tip location was displayed three-dimensionally on the monitor. The downside of this photograph depicted the cephalad side of the patient due to the operator standing in the cephalad position. 
Figure 3. Pedicle screw trajectory on the horizontal plane

Pedicle screw trajectory was measured as the angulation from sagittal line on the horizontal plane. Anatomical: Anatomical data was obtained from cadaver measurements reported by Panjabi et al. ${ }^{19}$; Computer-assisted: the angle in the computer-assisted navigation group; Manual: Manual data was obtained from fifty cases of previous operation using a conventional manual insertion technique. Asterisk indicates a significant difference from manual data at $\mathrm{P}<0.05$ level.

Figure 4. 17 years old male, Postlaminectomy kyphosis after posterior decompression for Chiari malformation.

A,B: Severe cervical kyphosis of 48 degrees from C2 to C5 with intractable neck pain C,D: Significant asymmetry in pedicle diameter and angle was demonstrated on CT images at C4 and C5 level (arrow).

E: Cervical pedicle screw fixation from C2 to C5 was successfully conducted with the assistance of navigation system. At one year postoperatively, bony fusion is complete without correction loss and instrumentation failure. The intractable neck pain disappeared. F: Intraoperative navigation image. The instrument tip was located in the middle of pedicle accurately shown on horizontal and pedicle-axis images. 
Figure 5. 52 years old male, Metastatic cervical spine tumor originated from prostate carcinoma.

A,B: Radiograph and MR scan demonstrated multiple bone metastases with kyphotic deformity. The spinal cord compression was depicted at C5-6 level. .

C, D, E: The posterior decompression and stabilization with CPS was performed with the use of navigation system followed by anterior decompression and fibula graft. The postoperative CT scans demonstrated the accurate screw placements.

Figure 6. 68 years old male, Cervical spondylotic myelopathy with kyphotic deformity and segmental instability at C3/4 level.

A,B: Severe cervical spondylosis with kyphosis of 22 degrees at C2-5. Spinal cord compression was depicted at C3/4 level.

C,D,E: Posterior correction and stabilization with CPS was performed at C3-5 followed by laminectomy at C3-7 level. Postoperative kyphosis was corrected to 6 degrees and maintained. Postoperative CT scan demonstrated the accurate placement of C5 screw in the narrow pedicle. Eighteen months radiograph showed a complete fusion.

F: Intraoperative navigation image. The instrument tip was located in the middle of pedicle accurately shown on horizontal and pedicle-axis images. 
Newly Developed Screw Insertion Guiding System for Cervical and Thoracic Pedicle Screw Insertion

\section{(Approved by Pharmaceutical Affairs Law, and New Design} for Practical Use in Japan)

\section{Screw Driver}

r

Awl

Probe

Screw Tap

$$
3.5-4.5
$$

$\mathrm{mm}$
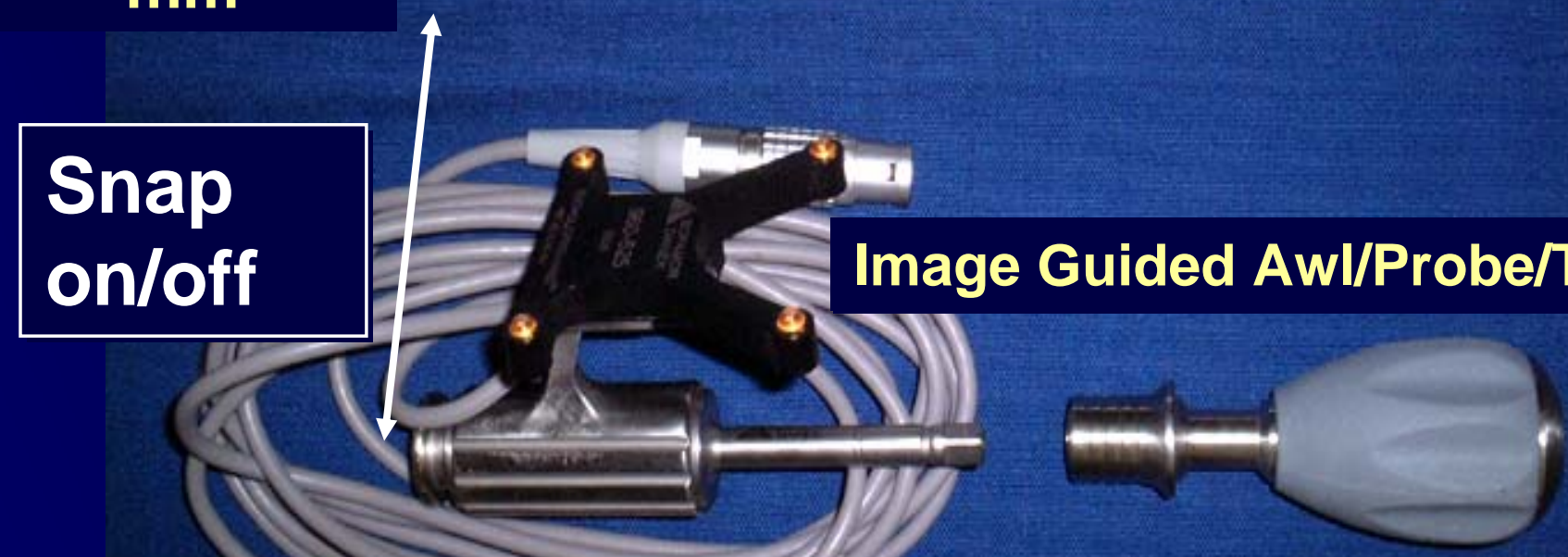
Screw Insertion with simultaneous and threedimensional instrument/screw tip information

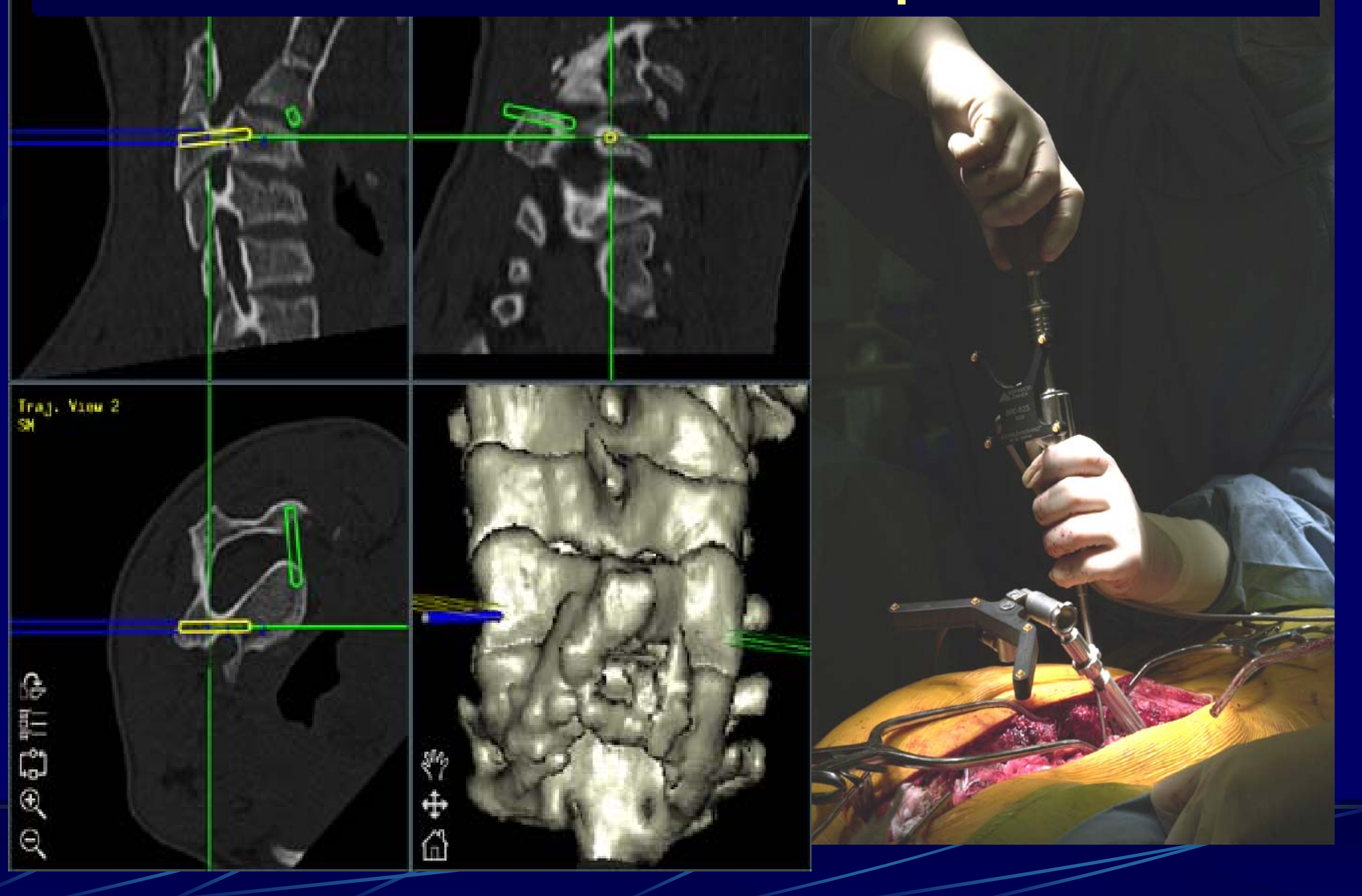




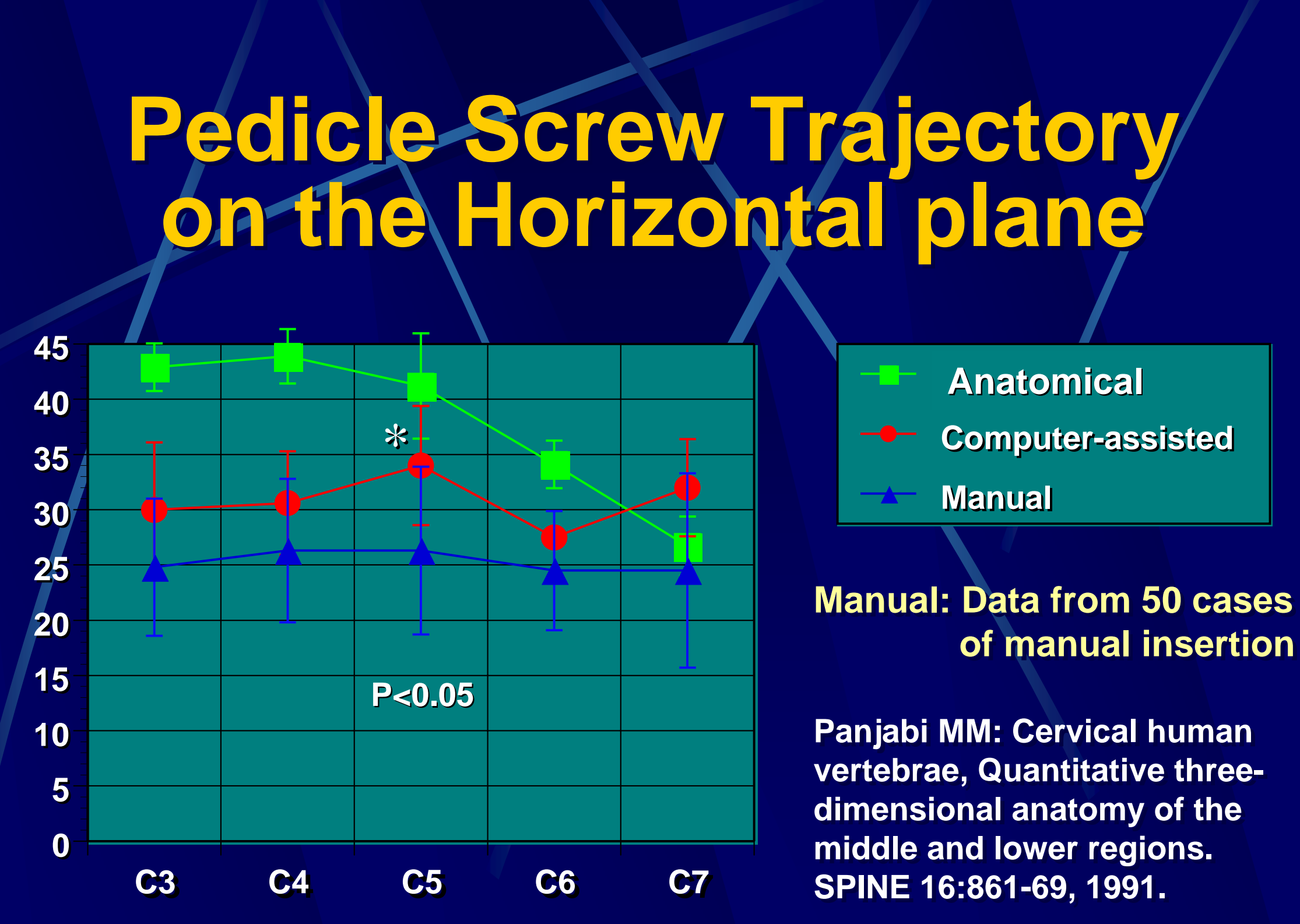


Postlaminectomy Kyphosis Following Posterior Decompression for Chiari Malformation
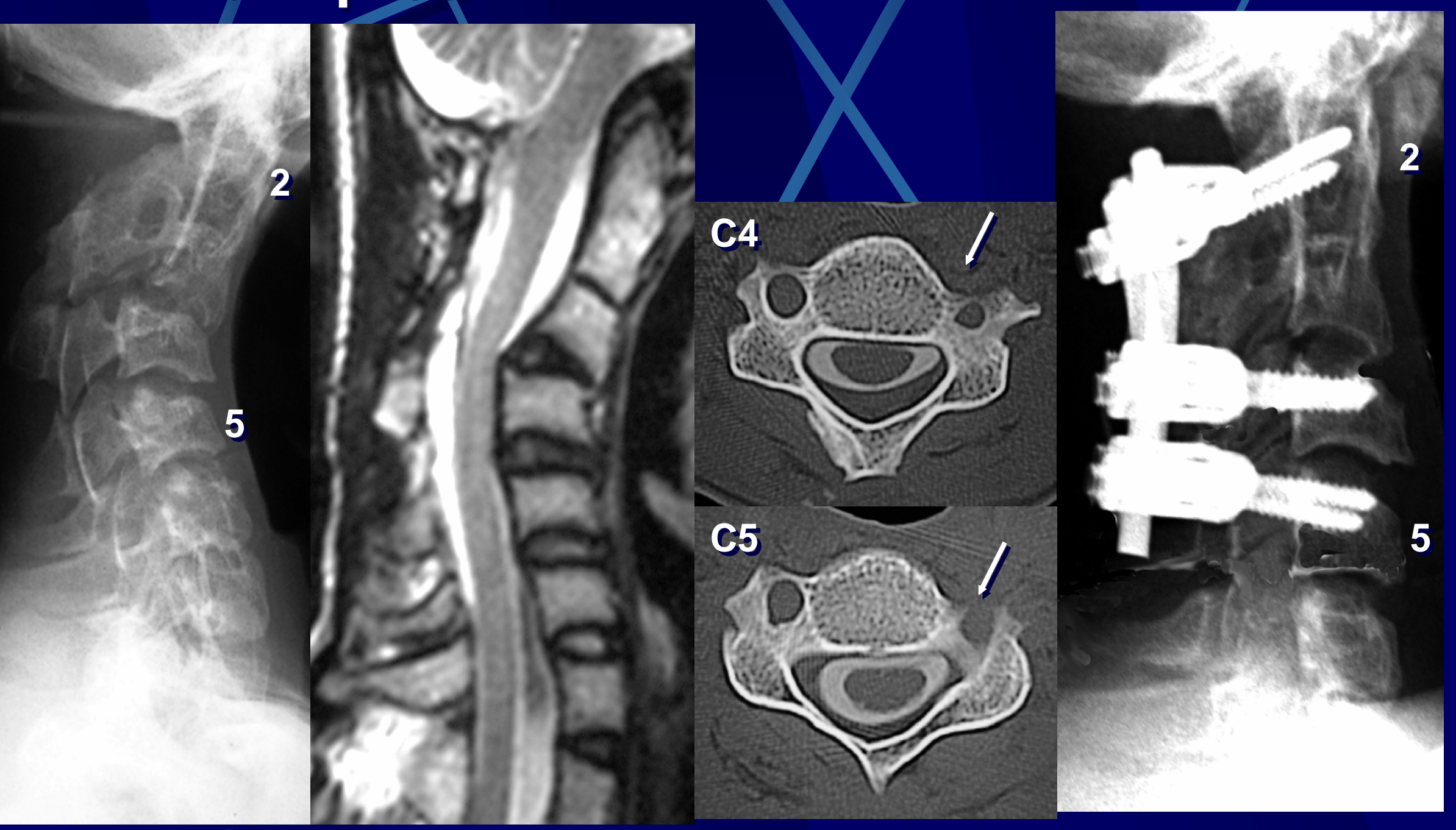


\section{Computer-assisted Screw Insertion in Adolescent Patient with Anomaly}

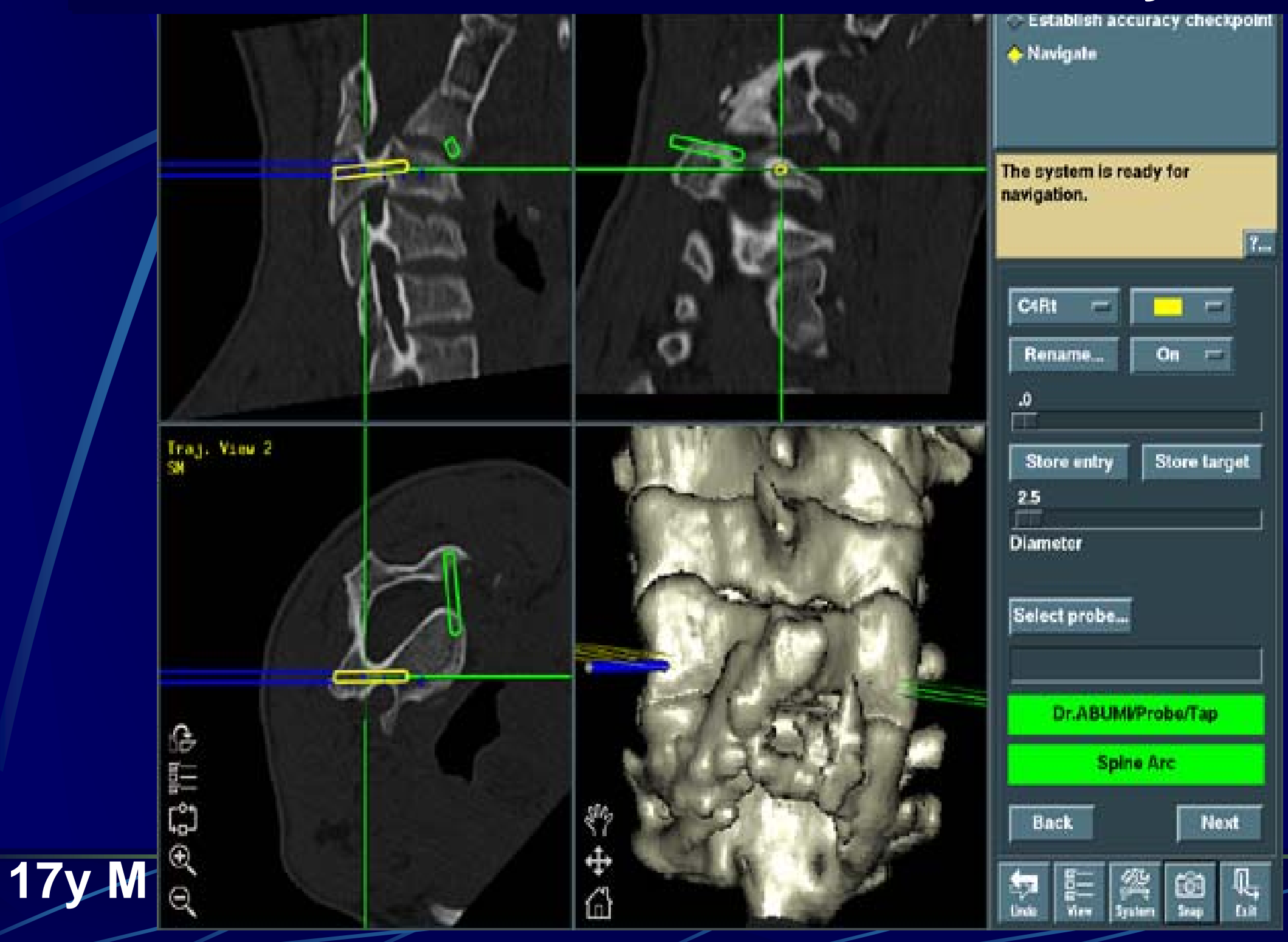


Cervical Spondylotic Myelopathy with Kyphotic Deformity
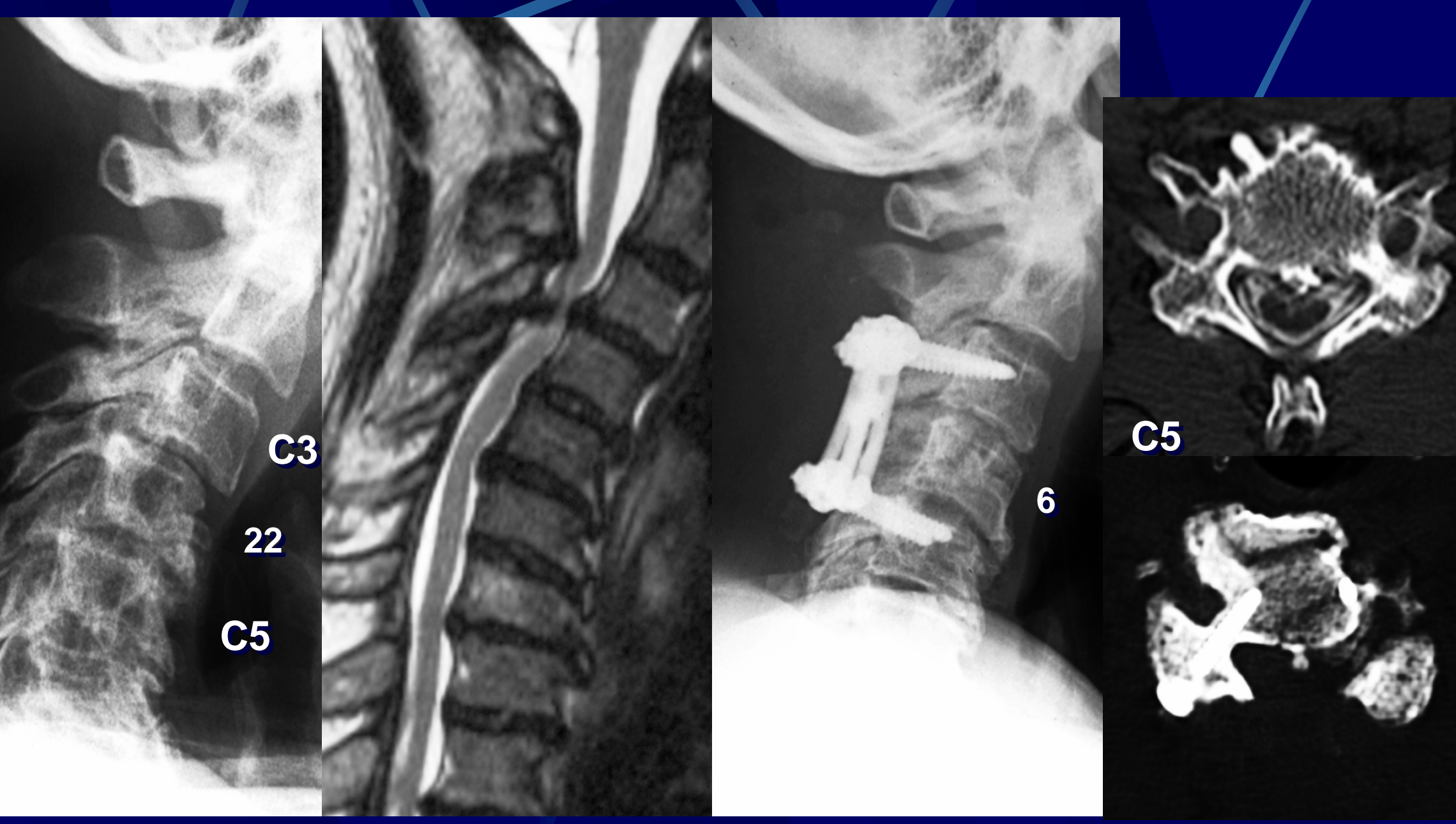

$68 \mathrm{y}$

Kyphosis and Segmental Instability at C3/4 


\section{Computer-assisted Screw Insertion in Severe Cervical Spondylosis}

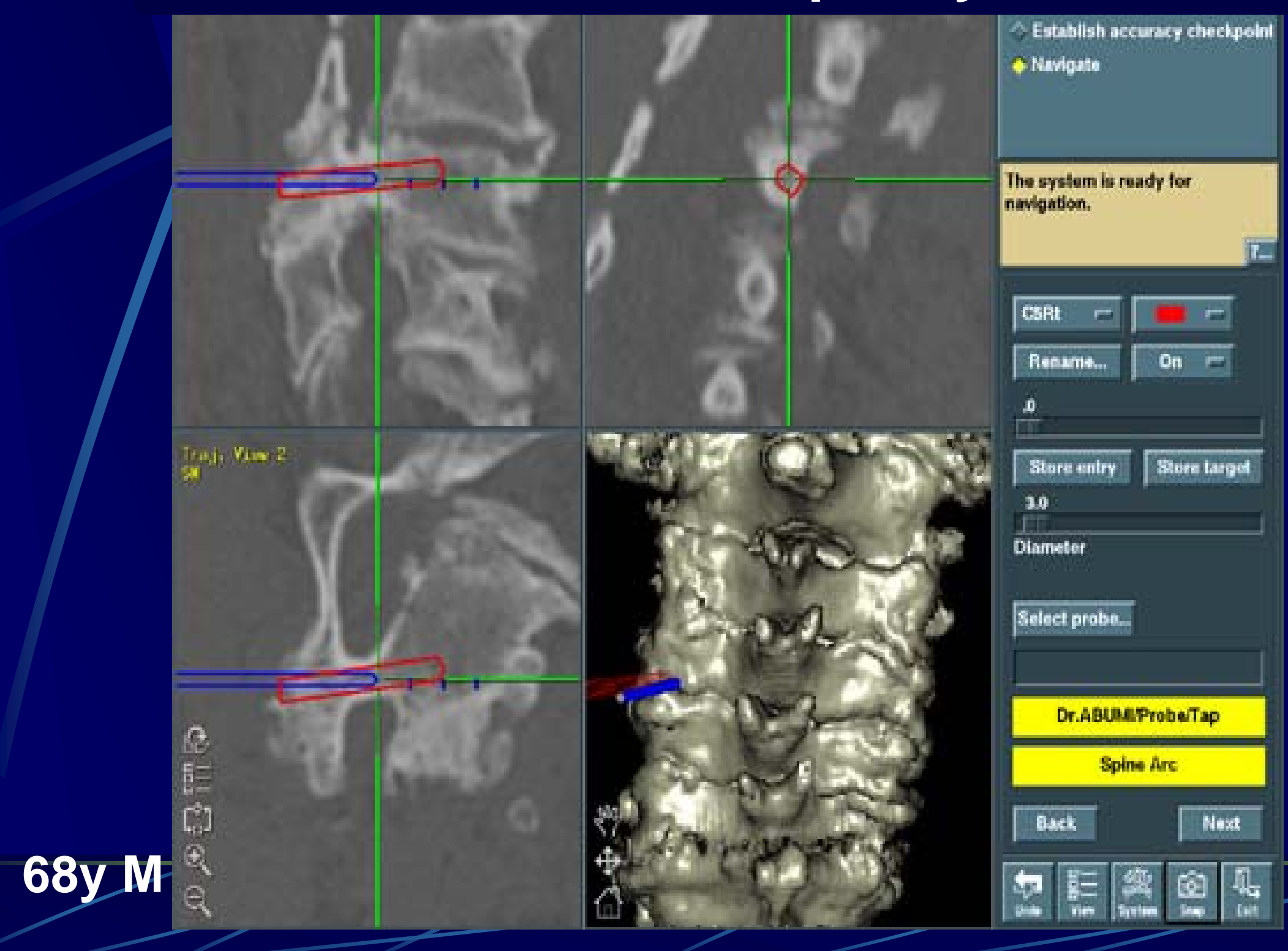




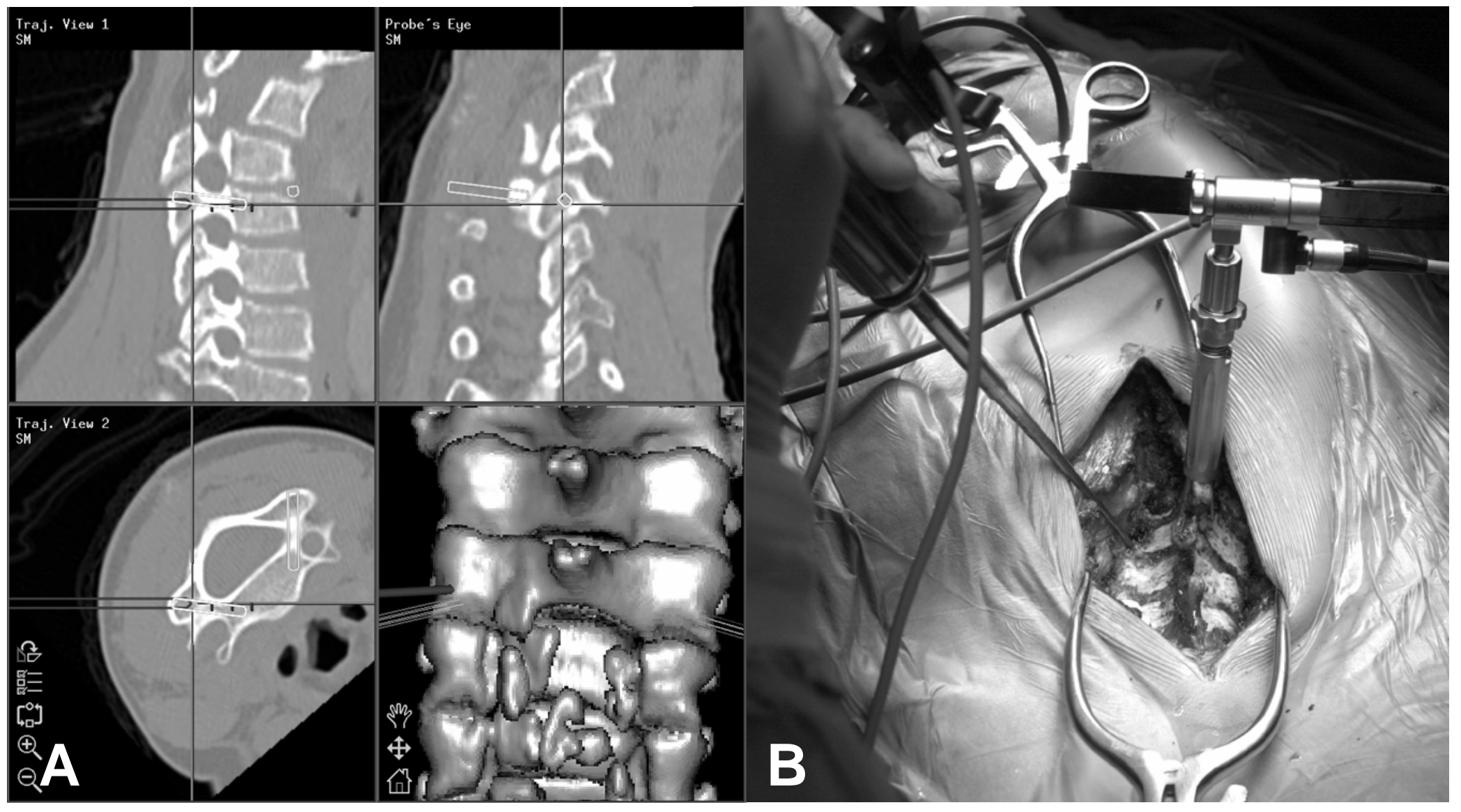




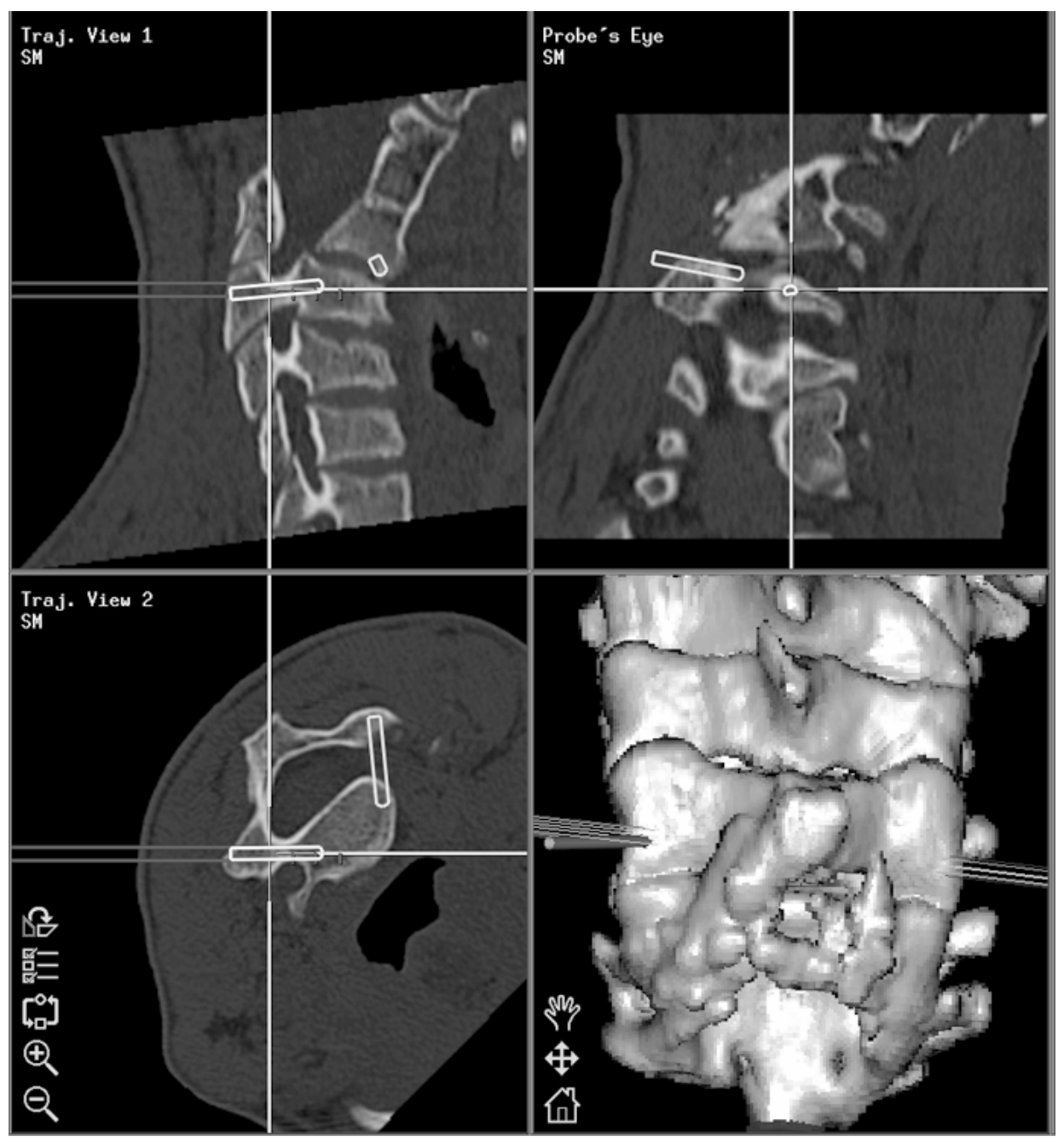




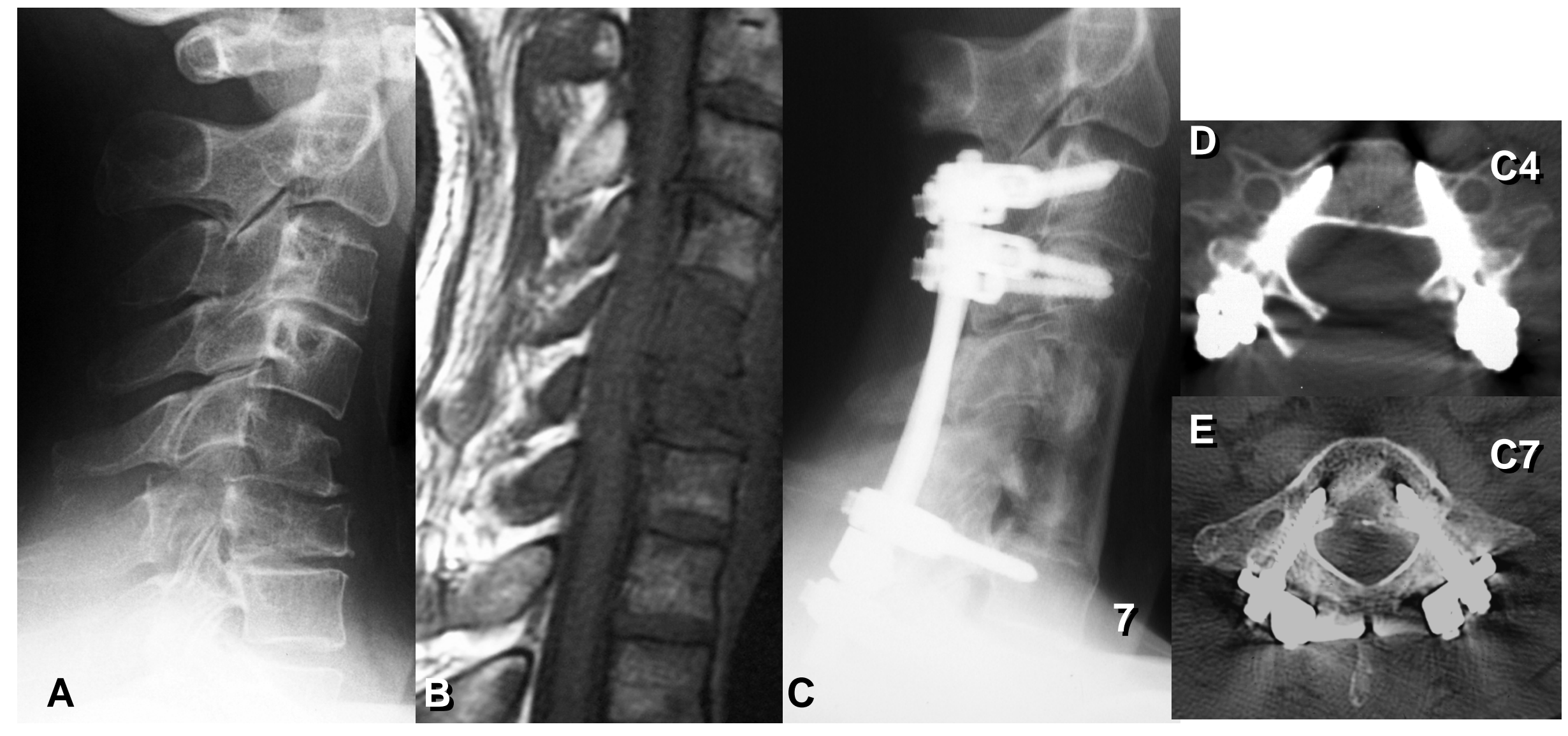

Figure 5 (A-E) 


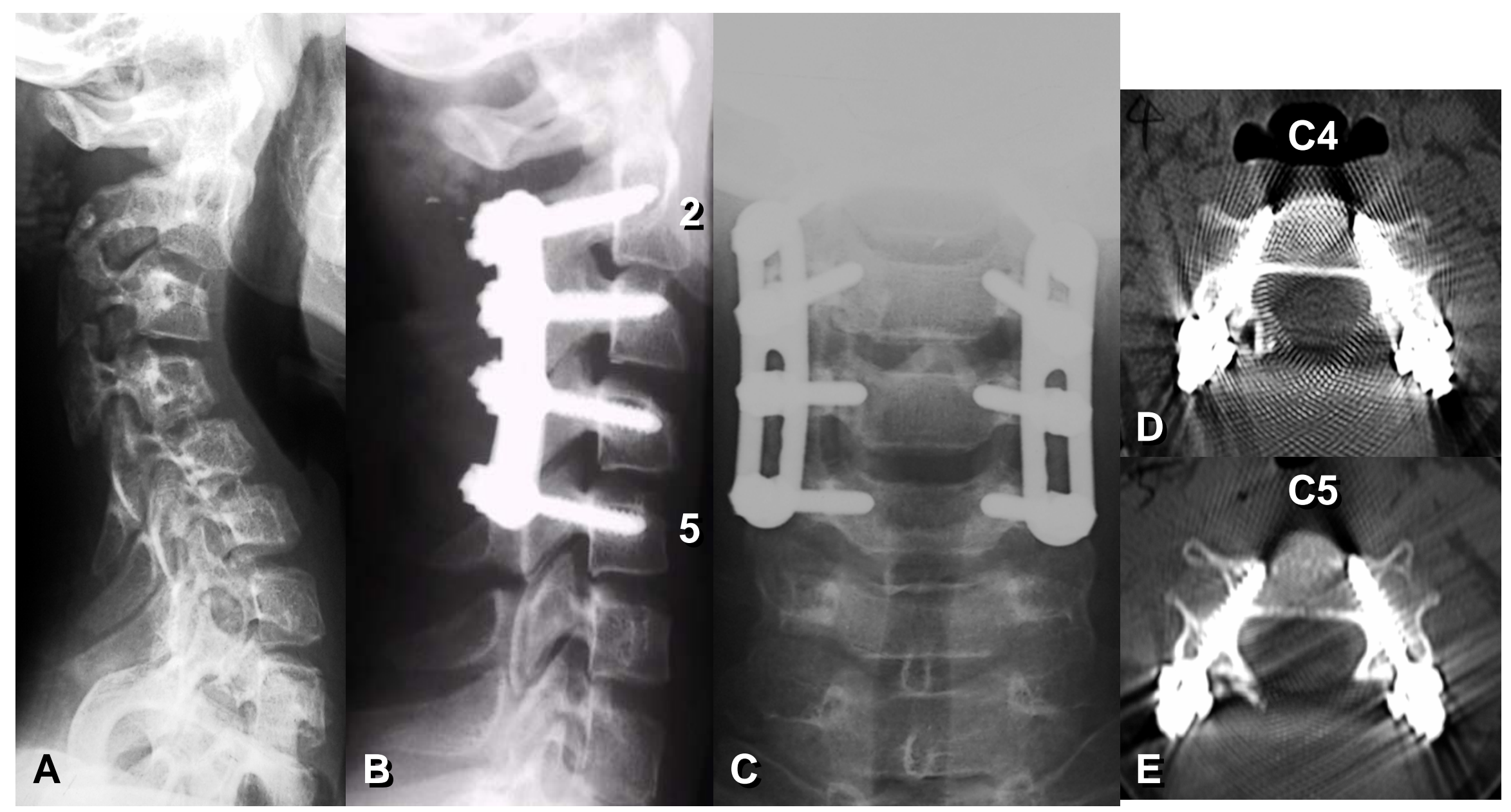

Figure 7 (A-E) 